CLINICAL STUDY

\title{
Risk factors for post-treatment hypogonadism in testicular cancer patients
}

\author{
Jakob Eberhard ${ }^{1,2}$, Olof Ståhl ${ }^{1,2}$, Magdalena Cwikiel ${ }^{1}$, Eva Cavallin-Ståhl ${ }^{1}$, Yvonne Giwercman ${ }^{3}$, \\ Eva Cecilia Salmonson ${ }^{4}$ and Aleksander Giwercman ${ }^{2,3}$ \\ ${ }^{1}$ Department of Oncology, Lund University Hospital, Lund University, SE 22185 Lund, Sweden, ${ }^{2}$ Reproductive Medicine Centre, Malmö University \\ Hospital, Lund University, SE 20502 Malmö, Sweden, ${ }^{3}$ Department of Clinical Sciences, Lund University, SE 20520 Malmö, Sweden and ${ }^{4}$ Department \\ of Radiology, Centre for Imaging and Physiology, Lund University Hospital, Lund University, SE 22185 Lund, Sweden \\ (Correspondence should be addressed to J Eberhard; Email: jakob.eberhard@med.lu.se)
}

\begin{abstract}
Objectives: Testicular germ-cell cancer (TGCC) patients are at risk of developing hypogonadism but no risk factors have yet been defined.

Methods: Blood was collected from 143 TGCC patients (after orchidectomy, prior to further therapy (T0) and 6, 12, 24, 36 and 60 months (T6, T12, T24, T36 and T60) after therapy). Biological hypogonadism (BH) was defined as: serum testosterone below $10 \mathrm{nmol} / \mathrm{l}$ and/or $\mathrm{LH}>10 \mathrm{IU} / \mathrm{l}$; odds ratios (ORs) for $\mathrm{BH}$ with $\mathrm{BH}$ at TO, age, stage of disease, testicular characteristics, and androgen receptor polymorphism as predictors were calculated as well as the OR for developing BH post-treatment (one to two cycles of adjuvant chemotherapy (ACT) versus three to four cycles of higher dose chemotherapy (HCT) versus adjuvant radiotherapy (RT)).

Results: HCT increased the OR for BH at T6 (OR 22, 95\% confidence interval (CI) 4.4-118) and T12 (OR $5.8,95 \%$ CI 1.5-22). RT increased the OR at T6 (OR 10, 95\% CI 2.1-47) and at T12 (OR 3.9, 95\% CI 1.1-14). Microlithiasis predicted BH at TO (OR 11, 95\% CI 1.2-112), T12 (OR 3.9, 95\% CI 1.1-13), T24 (OR 3.0, 95\% CI 1.0-8.8), T36 (OR 5.4, 95\% CI 1.7-17) and T60 (OR 4.4, 95\% CI 1.2-16). BH at T0 was a risk for BH at T6 (OR 53, 95\% CI 19-145), T12 (OR 125, 95\% CI 37-430), T24 (OR 88, 95\% CI 26-300) and T36 (OR 121, 95\% CI 32-460).

Conclusions: It is clinically relevant that $\mathrm{BH}$ at $\mathrm{TO}$ and testicular microlithiasis were predictive factors for post-treatment $\mathrm{BH}$. HCT and RT gave temporary $\mathrm{BH}$.
\end{abstract}

European Journal of Endocrinology 158 561-570

\section{Introduction}

Testicular germ-cell cancer (TGCC) patients are at risk of developing Leydig cell insufficiency $(1,2)$. Orchidectomized TGCC patients were reported to have subnormal testosterone values in contrast to orchidectomized noncancer patients (2), indicating that the disease per se is affecting the Leydig cell function. Different treatment modalities, such as orchidectomy, chemotherapy and radiotherapy may also affect the Leydig cell function, although the data are rather conflicting. Furthermore, although serum testosterone levels may decrease following orchidectomy (3), a compensatory, time-related improvement in Leydig cell function has been postulated (4). It may, therefore, imply that men being hypogonadal shortly after testis removal may recover and should, therefore, not be offered androgen replacement therapy immediately.

A Leydig cell impairment, with subnormal/normal s-testosterone values and/or increased luteinizing hormone (LH), was found in patients who had undergone chemotherapy (5-8). However, in other studies, no difference in testosterone or $\mathrm{LH}$ after chemotherapy was found $(9,10)$.
These discrepancies might be due to differences in the chemotherapy used, doses and also the length of followup time.

Many studies have focused on testosterone levels in men treated for TGCC, only few dealt with risk factors for development of hypogonadism. In a 10-year follow-up study, Nord et al. found that men treated for TGCC had a three- to fourfold risk to develop hypogonadism and that the risk increased with age and treatment intensity (11, 12). Another study reported increased risk of gonadal dysfunction in long-term survivors of testicular cancer and there was a dose-response effect following chemotherapy (12).

Our prospective longitudinal study was carried out to identify potential patient, disease and treatment-related factors associated with testicular dysfunction. Pretreatment hormone levels, age, stage of disease, treatment and contralateral testicular status in terms of volume, consistency and ultrasound pattern were investigated. Furthermore, polymorphic variations in the androgen receptor $(\mathrm{AR})$ gene, which have an impact on androgen sensitivity $(13,14)$ and therefore might influence the risk of developing hypogonadism, were also studied. 
We assessed the predictive value of above-mentioned characteristics in relation to risk for post-treatment biochemical signs of androgen deficiency.

\section{Patients and methods}

\section{Patients}

All patients with TGCC, who had been diagnosed after March 1996 at the Department of Oncology, Lund University Hospital and were under the age of 50, were eligible for the study (15). Six time points were defined for hormonal analysis: after orchidectomy, but before further treatment (T0) and 6,12, 24, 36 and 60 months (T6, T12, T24, T36 and T60) after completed therapy. Testosterone and LH were measured at each time point.

The patients were included from March 2001 until the end of the year 2005 and during this period, 200 men were eligible. Out of these, 28 denied to participate and 23 were excluded due to comorbidity. Of the remaining 149 men, 6 patients did not deliver any blood samples. From all other patients $(n=143)$, one or more blood samples were collected (Figs 1 and 2).

All participated with written informed consent according to protocols approved by the ethical review boards of Lund University.

\section{Cancer treatment}

Treatment was given according to the Swedish Norwegian Testicular Cancer Group (Swenoteca) protocols $(16,17)$.
The surveillance group (SO) consisted of 13 men, 5 with seminomas and 8 with non-seminomas. These men did not receive any further therapy after orchidectomy. Patients with clinical stage I (CSI) non-seminomas (NSGCT) were offered either adjuvant chemotherapy (ACT) or surveillance. Patients with CSI seminoma (SGCT) received adjuvant radiotherapy (RT) or chemotherapy (ACT) or entered a surveillance programme. Patients with more advanced disease were treated with chemotherapy. Standard chemotherapy of NSGCT was the BEP regimen (Bleomycin $30000 \mathrm{IU}$ day 1.5 and 15; Etoposide $100 \mathrm{mg} / \mathrm{m}^{2}$ day 1-5; Cisplatin $20 \mathrm{mg} / \mathrm{m}^{2}$ day $1-5$, given every third week). In six NSGCT patients given ACT, Etoposide was replaced with Vinblastin $0.15 \mathrm{mg} / \mathrm{kg}$, max $11 \mathrm{mg}$ /day, day 1-2 (CVB regimen). ACT for SGCT consisted of one to two cycles of Carboplatin, AUC 7.

ACT (BEP/CVB/Carboplatin) was given to 43 patients. Among these, 37 patients got one cycle and 6 got two cycles of chemotherapy. Forty-two patients with metastatic disease received three or four cycles of chemotherapy, defined as high dose CT (HCT). Among patients with NSGCT, 15 received three cycles and 19 received four cycles of BEP. Four cycles of EP (BEP minus Bleomycin) were given to eight patients with advanced SGCT. Nine patients (six NSGCT and three SGCT) received more intensive treatment (HDT).

Thirty-six patients with seminomas received adjuvant radiotherapy (RT). It was administered to para-aortic and ipsilateral iliac lymph nodes. A target dose of 25.2 Gy was given in 14 fractions. The total dose to the remaining testicle was estimated retrospectively to be $0.04-0.43 \mathrm{~Gy}$ in seven randomly selected men.

An overview of patient characteristics is presented in Table 1.

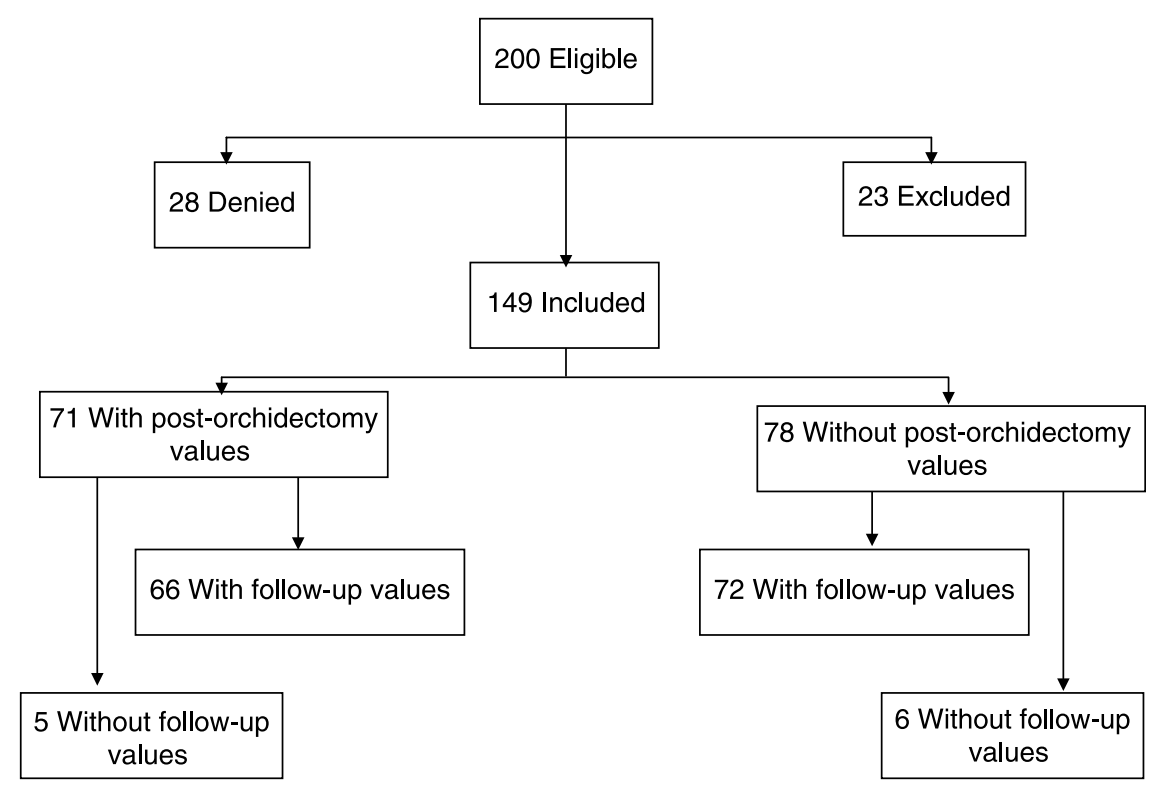

Figure 1 Flow chart describing the patient material: from six patients, we did not collect any samples; one did not come for follow-up, one moved to another region immediately after inclusion, three have not yet delivered their first sample after inclusion and one died of progressive disease. 


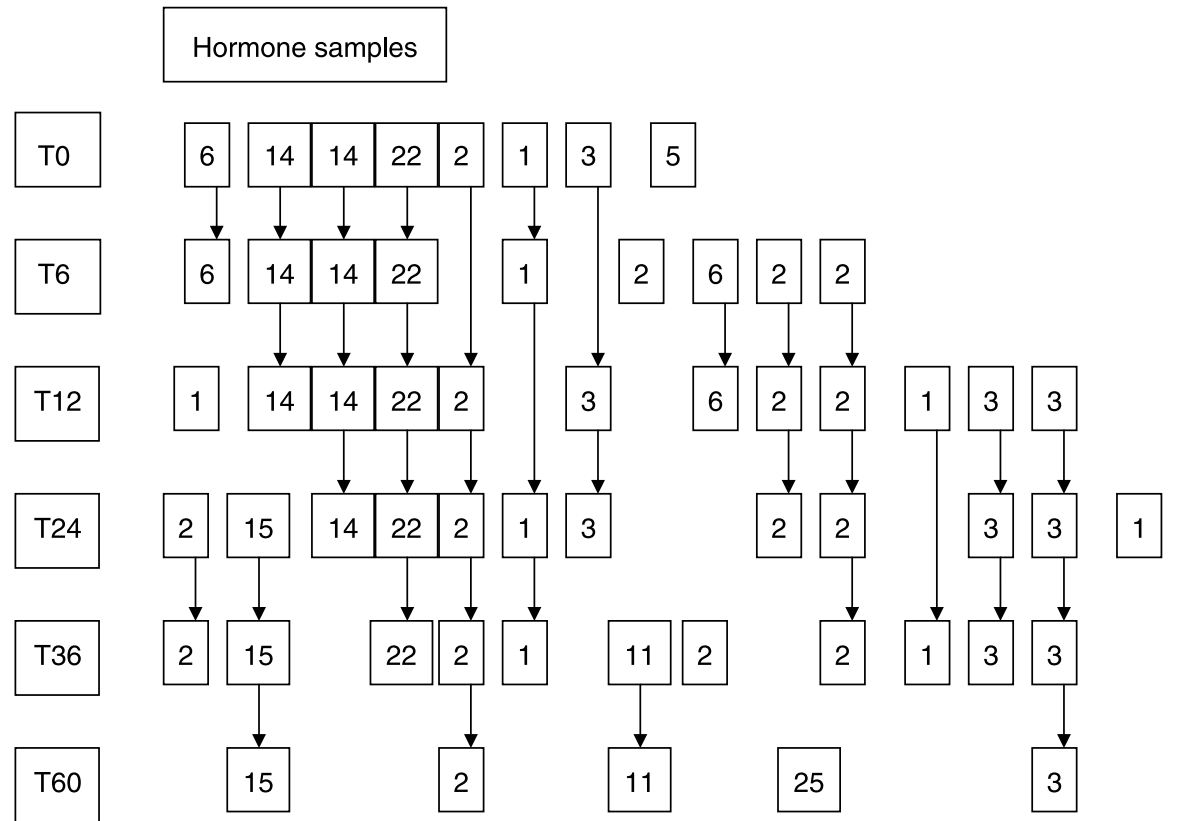

Figure 2 Flow chart describing the number of serum testosterone samples analysed. The arrows connect samples from the same patients. Post-orchidectomy samples (T0); T6, T12, T24, T36 and T60 indicate 6, 12 , 24, 36 and 60 months post-treatment. The figures differ slightly from those in Table 2 since nine patients with testosterone values have missing $\mathrm{LH}$ values and therefore cannot be grouped as hypogonadal.

\section{Hormone analysis}

Blood sampling was performed between 0800 and $1500 \mathrm{~h}$. During the study period, methodological changes of the hormonal analyses were made. Two methods of assessing serum values of testosterone were applied. Since May 2001, a luminometric technique has been applied (Access; Beckman Coulter, Chaska, MN, USA) and previously a RIA, an 'in-house' method developed at the department of clinical chemistry in Malmö, was used. RIA values were converted to Access (Access value $=1.19 \times$ RIA value $=-1.07$ ).

After 5 April 2000, LH in serum was determined with the Elecsys LH immunoassay (Roche Diagnostics). Before that date, LH was determined with an immunofluorometric assay (DELFIA LH, Wallac Oy, Turku, Finland). A conversion between the methods was hence necessary to carry out (Elecsys LH immunoassay value $=0.953 \times$
DELFIA value $=+0.602$ ). Furthermore, five LH samples were analysed in another laboratory using an immunofluorometric assay (Immuno1, Bayer AG). The conversion factor between the two laboratories was calculated based on parallel measurements done on serum from 30 healthy fishermen under the age of 50 (Elecsys LH immunoassay value $=1.79 \times$ Immuno1 value $=+0.05$ ).

In a pooled dataset, after conversion of the values, no difference in hormone levels was found when samples analysed by different methods were compared with each other.

\section{AR gene polymorphism analysis}

Blood samples for DNA analysis were available from 83 men. These subjects did not differ from the remaining 66 regarding age, disease, stage, histological type, treatment

Table 1 Characteristics of 143 patients with testicular germ-cell cancer, who have delivered at least one blood sample for hormonal analysis.

\begin{tabular}{|c|c|c|c|c|c|c|}
\hline & $\begin{array}{c}\text { No therapy after } \\
\text { orchidectomy }\end{array}$ & ACT & НСТ & HDT & RT & Total \\
\hline No. of patients & 13 & 43 & 42 & 9 & 36 & 143 \\
\hline Age (median) ${ }^{\mathrm{a}}$ & 29 & 29 & 27 & 29 & 35.5 & 30 \\
\hline Seminoma & 5 & 2 & 8 & 3 & 36 & 54 \\
\hline Non-seminoma & 8 & 41 & 34 & 6 & 0 & 89 \\
\hline Stage I & 13 & 43 & $9^{b}$ & $2^{c}$ & 36 & 103 \\
\hline Stage II & 0 & 0 & 21 & 2 & 0 & 23 \\
\hline Stage III & 0 & 0 & 4 & 2 & 0 & 6 \\
\hline Stage IV & 0 & 0 & 8 & 3 & 0 & 11 \\
\hline
\end{tabular}

Royal Marsden Hospital (RMH) staging system has been used (30). ACT, one to two cycles of adjuvant chemotherapy; HCT, three to four cycles of chemotherapy; HDT, more intense treatment, more than four cycles of chemotherapy; RT, adjuvant radiotherapy.

${ }^{a}$ At the time of diagnosis.

${ }^{\mathrm{b}}$ Recurrent disease including four patients with marker elevation.

${ }^{\mathrm{c}}$ Recurrent disease. 
or sperm concentration at T0 (data not shown). Genomic DNA was prepared from peripheral leukocytes, and the CAG (83 subjects) and GGN repeats of the AR gene (81 subjects) were amplified by PCR and subsequently analysed externally on a Beckman Coulter CEQ 2000XL (Beckman Coulter) sequencing gear, as previously described (18).

\section{Ultrasound examination}

Of the 143 patients, 107 subjects underwent an ultrasound of the remaining testicle between 6 and 12 months after inclusion, performed by the same radiologist. The outcome was presence or absence of microlithiasis, defined as at least five uniform, non-shadowing echogenic foci of 1-3 mm, scattered throughout the testicular parenchyma (19, 20) (Fig. 7). For determination of testicular volume, we used the formula for the volume of ellipsoid: $1 / 3 \times \pi \times$ length $\times$ wide $\times$ thickness $\times 1 / 2(21)$. The consistency was investigated by palpation and assessed as being normal or soft.

\section{Statistical analysis}

Statistical analyses were performed using SPSS 11.0 software (Chicago, IL, USA).

Regarding hypogonadism at TO, patients being hCG positive at this time point were excluded from the statistical analysis. Seven patients receiving testosterone replacement were considered hypogonadal from the time the replacement was given. Two of them were in the ACT group, two in the HCT group and two in the RT group. The last one was in the group receiving more intensive treatment (more than four cycles of chemotherapy).

Biochemical hypogonadism $(\mathrm{BH})$ was defined as s-testosterone $<10 \mathrm{nmol} / \mathrm{l}$ and/or s-LH $\geq 10 \mathrm{IU} / \mathrm{l}$ (22).

Since the blood samples were taken between 0900 and $1500 \mathrm{~h}$ and the levels of testosterone but not LH decrease during the day, for all statistically significant findings the possible associations were re-tested with $\mathrm{LH}>10 \mathrm{IU} / \mathrm{l}$ as the only indicator of $\mathrm{BH}$.

Primarily, using binary logistic regression analysis, the odds ratio (OR) for $\mathrm{BH}$ was calculated for TGCC patients using the ACT group as a reference group. A separate comparison was done for each treatment modality (HCT/RT) and post-treatment time point. Furthermore, the risk for the whole group of TGCC men for developing $\mathrm{BH}$ was tested at different post-treatment time points, with respect to the following potential predictive factors: $\mathrm{BH}$ at TO (yes/no), AR CAG $(<21,21-22,>22)$ and GGN $(<23,23,>23)$ repeat number, stage of disease (I-IV), testicular consistency (normal/soft), ultrasound ( $+/-$ microlithiasis), volume of the contralateral testis $(<15$ vs $\geq 15 \mathrm{ml})$ and age $(<30$ vs $\geq 30$ years $)$. Patients presenting with microlithiasis were complementary analysed after excluding those who were hypogonadal at $\mathrm{TO}$. The method of hormone analysis was used as potential confounder.
All analyses were performed separately for each time point.

$P<0.05$ was considered as statistically significant.

\section{Results}

\section{Absolute hormone levels}

The absolute testosterone and LH values with medians and ranges are measured for all different therapy arms and at all the different follow-up time points (Tables 3 and 4).

\section{$B H$ in relation to treatment}

For the HCT group, a significantly increased OR for hypogonadism, when compared with the ACT group, was found after 6 (OR: 22, 95\% confidence interval (CI) 4.4-118) and 12 (OR 5.8, 95\% CI 1.5-22) months post-therapy. Also in the RT group, a significantly increased OR was observed when compared with the ACT group after 6 (OR 10, 95\% CI 2.1-47) and after 12 (OR 3.9, 95\% CI 1.1-14) months post-therapy. The number of patients from both surveillance and the HDT group were too few to analyse (Figs 3 and 4).

\section{Post-treatment BH in relation to $B H$ at $\mathrm{TO}$}

$\mathrm{BH}$ at T0 strongly predicted $\mathrm{BH}$ at T6 (OR 53, 95\% CI 19145), T12 (OR 125, 95\% CI 37-430), T24 (OR 88, 95\% CI 26-300) and T36 (OR 121, 95\% CI 32-460). At TO, 22 men were hypogonadal. After 1 year, 12 out of 20 and after 2 years 6 out of 11 had normal hormone levels of those 22 initially being hypogonadal (Table 2 and Fig. 5).

\section{$B H$ in relation to stage, age and $A R$ polymorphisms}

No statistical significance was observed for stage of disease or age. AR polymorphisms of CAG and GGN repeat did not have any effect on the risk of developing $\mathrm{BH}$.

\section{BH in relation to testicular characteristics}

Pathological ultrasound with presence of microlithiasis was predictive for $\mathrm{BH}$ at these time points: TO (OR 11, 95\% CI 1.2-112), T12 (OR 3.9, 95\% CI 1.1-13), T24 (OR 3.0, 95\% CI 1.0-8.8), T36 (OR 5.4, 95\% CI 1.717) and T60 (OR 4.4, 95\% CI 1.2-16; Fig. 6). When excluding patients being hypogonadal at TO a similar trend was observed, reaching statistical significance at T36 (OR 5.2, 95\% CI 1.5-19) and T60 (OR 4.7, 95\% CI 1.0-21). Testicular volume or consistency of the contralateral testicle was not associated with any increased risk for $\mathrm{BH}$.

For all above-mentioned statistically significant associations, the same trends were found when $\mathrm{LH}>$ $10 \mathrm{IU} / \mathrm{l}$ was used as the only indicator of BH (Fig. 7). 


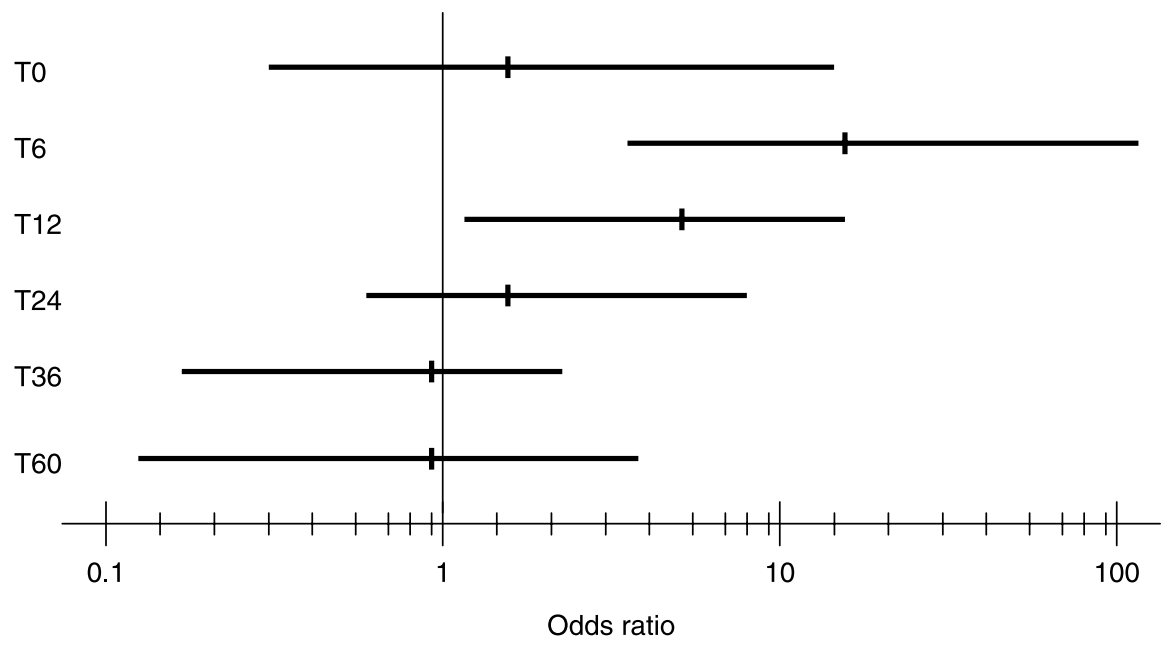

Figure 3 Therapy-dependent odds ratios of developing biological hypogonadism with the group given adjuvant chemotherapy as reference in patients treated with three to four cycles of chemotherapy. Post-orchidectomy samples (T0); T6, T12, T24, T36 and T60 indicate 6, 12, 24, 36 and 60 months post-treatment.

\section{Discussion}

We found microlithiasis in the remaining testis as significant risk factors for $\mathrm{BH}$, defined as low total testosterone and/or high LH levels in serum. In addition, BH prior to chemo- or radiotherapy and treatment modality seems to play a role in this context, higher doses of chemotherapy as well as adjuvant radiation being the most harmful to Leydig cells.

The issue of testosterone levels in men treated for TGCC was previously addressed by others (23-25). However, these studies did not clearly discriminate between treatment modalities and/or the post-treatment investigations were not performed at specific time points, allowing for more precise mapping of the process of Leydig cell recovery (Tables 3 and 4).

A significant increase in the OR for $\mathrm{BH}$ was observed in patients receiving three to four cycles of chemotherapy 6 and 12 months post-treatment. The same observation was made in the RT group. The choice of reference group was made on the assumption that one to two cycles of BEP only has limited impact on Leydig cell function. Another option had been to use the TO group or surveillance patients. However, with respect to T0, possible post-orchidectomy compensatory improvements of Leydig cell function and also possible temporary dysfunction after orchidectomy could not be taken into consideration. The number of surveillance patients was not sufficient to be used as reference and if men without TGCC were used as controls, the impact of therapy could not be discriminated from the negative effect of TGCC per se on Leydig cell function.

We have shown that Leydig cell function may occur over time, since no difference between the therapy groups was observed at T24 or later. This phenomenon is similar to that recently reported for sperm production, with a significant decline in sperm concentration after 6-12 months and after subsequent recovery to the pretreatment level (15). The time-related post-treatment recovery of Leydig cell function is in discordance with the data published by Nord et al. who reported an increased risk of hypogonadism 10 years after completion of TGCC treatment (11). However, the longer observation time and

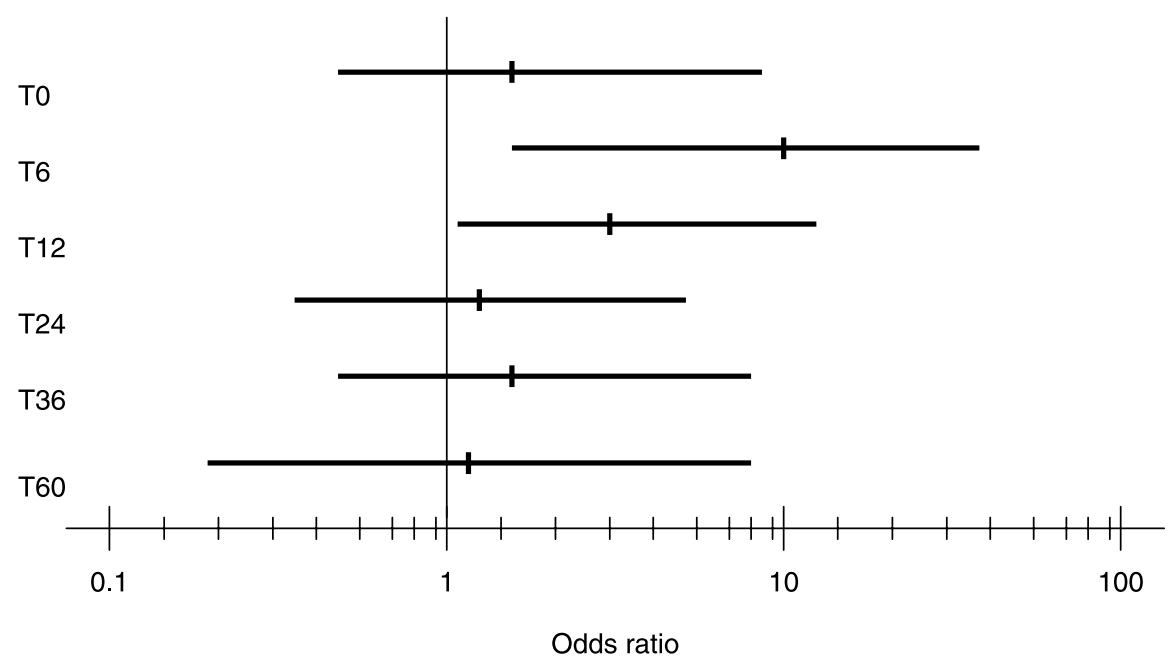

Figure 4 Therapy-dependent odds ratios of developing biological hypogonadism with the group given adjuvant chemotherapy as reference in patients treated with radiotherapy. Post-orchidectomy samples (T0); T6, T12, T24, T36 and T60 indicate 6, 12, 24,36 and 60 months post-treatment. 
Table 2 Characteristics of the 143 testicular germ-cell cancer (TGCC) patients included in the study, with and without biochemical signs of hypogonadism.

\begin{tabular}{|c|c|c|c|c|c|c|}
\hline & \multicolumn{6}{|c|}{ No. hypogonadal/all (\% hypogonadal) } \\
\hline & T0 & T6 & $\mathrm{T} 12$ & T24 & T36 & T60 \\
\hline \multicolumn{7}{|l|}{ Age, years } \\
\hline$<30$ & 9/32 (28\%) & $12 / 27(44 \%)$ & 13/32 (41\%) & $14 / 39(36 \%)$ & $14 / 33(42 \%)$ & $10 / 38(26 \%)$ \\
\hline$\geq 30$ & $21 / 44(48 \%)$ & $22 / 44(50 \%)$ & $22 / 42(52 \%)$ & $15 / 32(47 \%)$ & $13 / 31(42 \%)$ & $6 / 24(25 \%)$ \\
\hline \multicolumn{7}{|l|}{ Stage } \\
\hline 1 & $24 / 56(43 \%)$ & $21 / 54(39 \%)$ & $26 / 58(45 \%)$ & $19 / 54(35 \%)$ & $23 / 47(49 \%)$ & $12 / 39(31 \%)$ \\
\hline II & $4 / 11(36 \%)$ & $6 / 8(75 \%)$ & $5 / 9(56 \%)$ & $6 / 10(60 \%)$ & $3 / 12(25 \%)$ & $3 / 11$ (27\%) \\
\hline III & $1 / 2(50 \%)$ & $2 / 3(67 \%)$ & $1 / 2(50 \%)$ & $0 / 1(0 \%)$ & $0 / 1(0 \%)$ & $0 / 3(0 \%)$ \\
\hline IV & $1 / 7(14 \%)$ & $5 / 6(83 \%)$ & $3 / 5(60 \%)$ & $4 / 6(67 \%)$ & $1 / 4(25 \%)$ & $1 / 3(33 \%)$ \\
\hline \multicolumn{7}{|c|}{ Hypogonadal at T0 } \\
\hline Yes & $22 / 22(100 \%)$ & $9 / 21(43 \%)$ & $12 / 20(60 \%)$ & $6 / 11(55 \%)$ & $3 / 7(43 \%)$ & $0 / 0$ \\
\hline No & $0 / 36(0 \%)$ & $8 / 29(28 \%)$ & $6 / 26(23 \%)$ & $10 / 26(38 \%)$ & $9 / 15(60 \%)$ & $2 / 3(67 \%)$ \\
\hline \multicolumn{7}{|c|}{ No. of CAG repeats } \\
\hline$<21$ & $4 / 11(36 \%)$ & $3 / 7(43 \%)$ & $7 / 12(58 \%)$ & $7 / 16(44 \%)$ & $11 / 20(55 \%)$ & $5 / 19(26 \%)$ \\
\hline $21-22$ & $3 / 12(25 \%)$ & 4/11 (36\%) & 4/12 (33\%) & $11 / 18(61 \%)$ & 9/18 (50\%) & $5 / 10(50 \%)$ \\
\hline$>22$ & $6 / 15(40 \%)$ & $5 / 13(38 \%)$ & $6 / 15(40 \%)$ & $4 / 19(21 \%)$ & $4 / 16(25 \%)$ & $4 / 14(29 \%)$ \\
\hline \multicolumn{7}{|c|}{ No. of GGN repeats } \\
\hline$<23$ & $1 / 4(25 \%)$ & $1 / 2(50 \%)$ & $0 / 3(0 \%)$ & $0 / 5(0 \%)$ & $2 / 6(33 \%)$ & $1 / 6(17 \%)$ \\
\hline$=23$ & $10 / 25(40 \%)$ & $10 / 23(43 \%)$ & $15 / 26(58 \%)$ & $15 / 31(48 \%)$ & $15 / 30(50 \%)$ & $7 / 16(44 \%)$ \\
\hline$\geq 23$ & $2 / 9(22 \%)$ & $1 / 6(17 \%)$ & $2 / 10(20 \%)$ & $7 / 17(41 \%)$ & $7 / 18(39 \%)$ & $6 / 19(32 \%)$ \\
\hline \multicolumn{7}{|l|}{ Microlithiasis } \\
\hline Yes & $14 / 32(44 \%)$ & $12 / 29(41 \%)$ & $17 / 28(61 \%)$ & 19/32 (59\%) & $19 / 29(66 \%)$ & $11 / 23(48 \%)$ \\
\hline No & $3 / 20(15 \%)$ & $9 / 18(50 \%)$ & $8 / 24(33 \%)$ & $9 / 26(35 \%)$ & $7 / 27$ (26\%) & $5 / 29(17 \%)$ \\
\hline \multicolumn{7}{|c|}{ Testicular consistence } \\
\hline Soft & $3 / 4(75 \%)$ & $2 / 3(67 \%)$ & $3 / 5(60 \%)$ & $2 / 5(40 \%)$ & $1 / 4(25 \%)$ & $1 / 3(33 \%)$ \\
\hline Normal & $26 / 71(37 \%)$ & $31 / 67(46 \%)$ & $31 / 68(46 \%)$ & $27 / 66(41 \%)$ & $26 / 59(44 \%)$ & $15 / 52(29 \%)$ \\
\hline \multicolumn{7}{|l|}{ Volume } \\
\hline$<15 \mathrm{ml}$ & $12 / 31(39 \%)$ & $14 / 28(50 \%)$ & $16 / 32(50 \%)$ & $18 / 33(55 \%)$ & $13 / 33(39 \%)$ & 9/25 (36\%) \\
\hline$\geq 15 \mathrm{ml}$ & $3 / 18(17 \%)$ & $5 / 16(31 \%)$ & $7 / 18(39 \%)$ & $8 / 24(33 \%)$ & $13 / 22(59 \%)$ & $7 / 25(28 \%)$ \\
\hline \multicolumn{7}{|l|}{ Treatment } \\
\hline Surveillance & 1/3 (33\%) & $5 / 7$ (71\%) & $3 / 5(60 \%)$ & $1 / 4(25 \%)$ & $2 / 4(50 \%)$ & $3 / 4$ (75\%) \\
\hline ACT & $9 / 27(33 \%)$ & $3 / 24(12 \%)$ & $6 / 25(24 \%)$ & $8 / 27(30 \%)$ & $9 / 23(39 \%)$ & $3 / 14(21 \%)$ \\
\hline НCT & $7 / 21(33 \%)$ & $13 / 18(72 \%)$ & $12 / 18(67 \%)$ & $9 / 18(50 \%)$ & $7 / 19(37 \%)$ & $4 / 20(20 \%)$ \\
\hline RT & $11 / 20(55 \%)$ & $10 / 18(56 \%)$ & $12 / 22(55 \%)$ & $7 / 17(41 \%)$ & $8 / 14(57 \%)$ & $5 / 17(29 \%)$ \\
\hline HDT & $2 / 5(40 \%)$ & $3 / 4(75 \%)$ & $2 / 4(50 \%)$ & $4 / 5(80 \%)$ & $1 / 4(25 \%)$ & $1 / 1(100 \%)$ \\
\hline
\end{tabular}

$\mathrm{ACT}$, one to two cycles of chemotherapy; HCT, three to four cycles of chemotherapy; RT, radiotherapy; HDT, more than four cycles of chemotherapy; RMH Royal Marsden Hospital staging system has been used (30).

therefore an age-dependent deterioration cannot be excluded in the latter study.

Although stage of disease and treatment are closely related, we did not find any association between disease stage and the risk of $\mathrm{BH}$. However, stage I patients can either be treated by ACT or by RT that are modalities apparently differing in their impact on Leydig cell function.

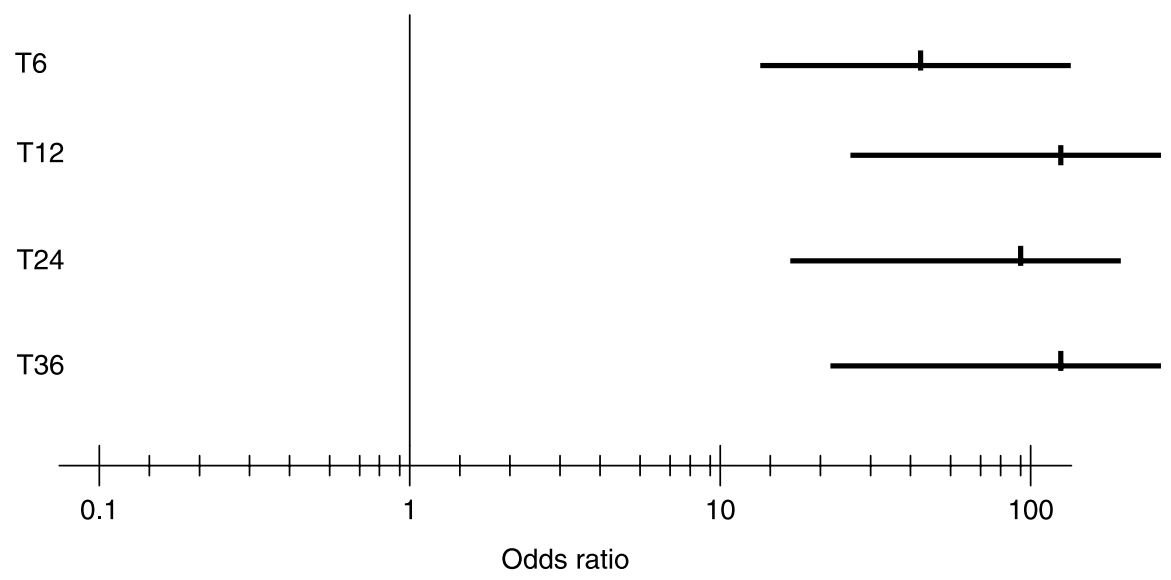

Figure 5 Odds ratios for biological hypogonadism for TGCC patients in relation to biological hypogonadism at T0. Post-orchidectomy samples (T0); T6, T12, T24 and T36 indicate 6, 12, 24 and 36 months post-treatment. After 60 months, statistical analysis was not done due to insufficient number of subjects. 


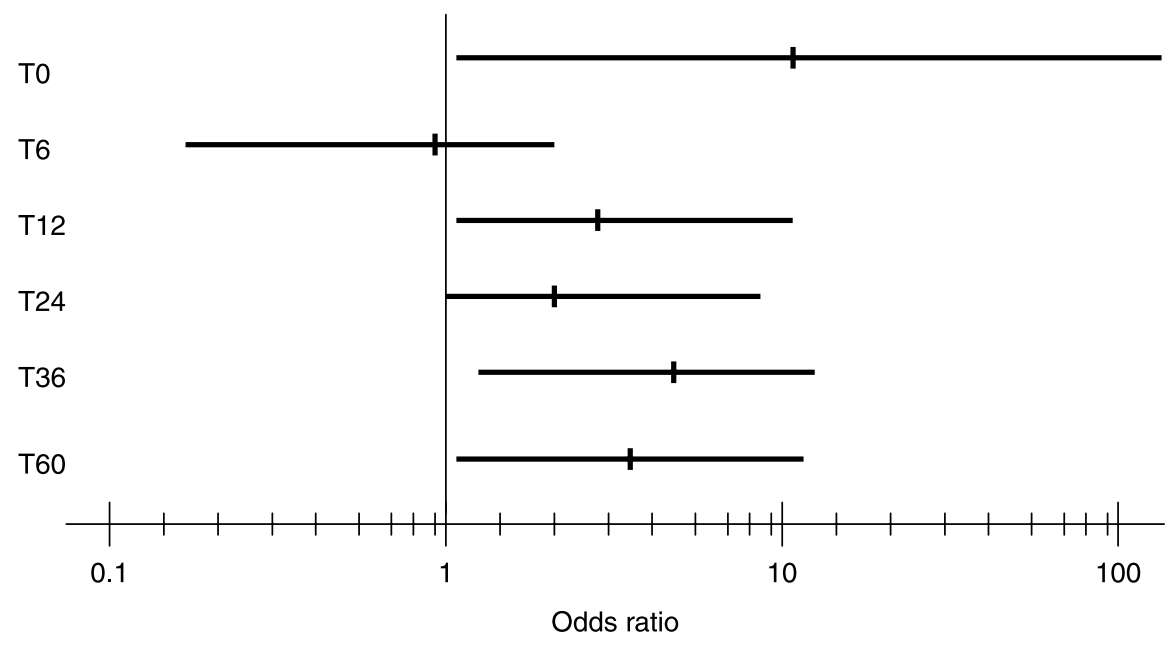

Figure 6 Odds ratios for biological hypogonadism in relation to microlithiasis of the remaining testicle. Postorchidectomy samples (T0); T6, T12, T24, T36 and T60 indicate 6, 12, 24, 36 and 60 months post-treatment.
Scrotal ultrasound is routinely used in the diagnosis of TGCC. Ultrasonically detected microlithiasis in the contralateral testis was associated with post-treatment risk of $\mathrm{BH}$ with an OR of 3-11. Microlithiasis has earlier been reported in patients with testicular dysgenesis syndrome (TDS) (26) and is believed to be associated with confirmed testicular cancer (27). Since Leydig cell dysfunction is considered as a part of the TDS, our results could support this observation. An increased risk of posttreatment hypogonadism in men with microlithiasis could be due to significant risk of androgen deficiency prior to chemo- or radiotherapy, which is also found to be a predictor of $\mathrm{BH}$ at $\mathrm{T} 6$ and at subsequent time points. However, microlithiasis remained a predictor of $\mathrm{BH}$ after exclusion of the subgroup of men who were hypogonadal at TO. To our knowledge, there are no studies indicating that cancer therapy can induce microlithiasis and therefore we assume that the pathological ultrasonic pattern was also present prior to treatment.

Sensitivity to androgens is genetically determined by the polymorphisms in the AR gene. Lower androgen sensitivity, with higher LH levels, was found in men with long CAG tracts (28). It could be anticipated that the risk of hypogonadism is modified by the length of this repeat. However, we found no association between any of the AR polymorphisms and biochemically defined hypogonadism. Similarly, no significant associations were found for testicular volume or consistency.

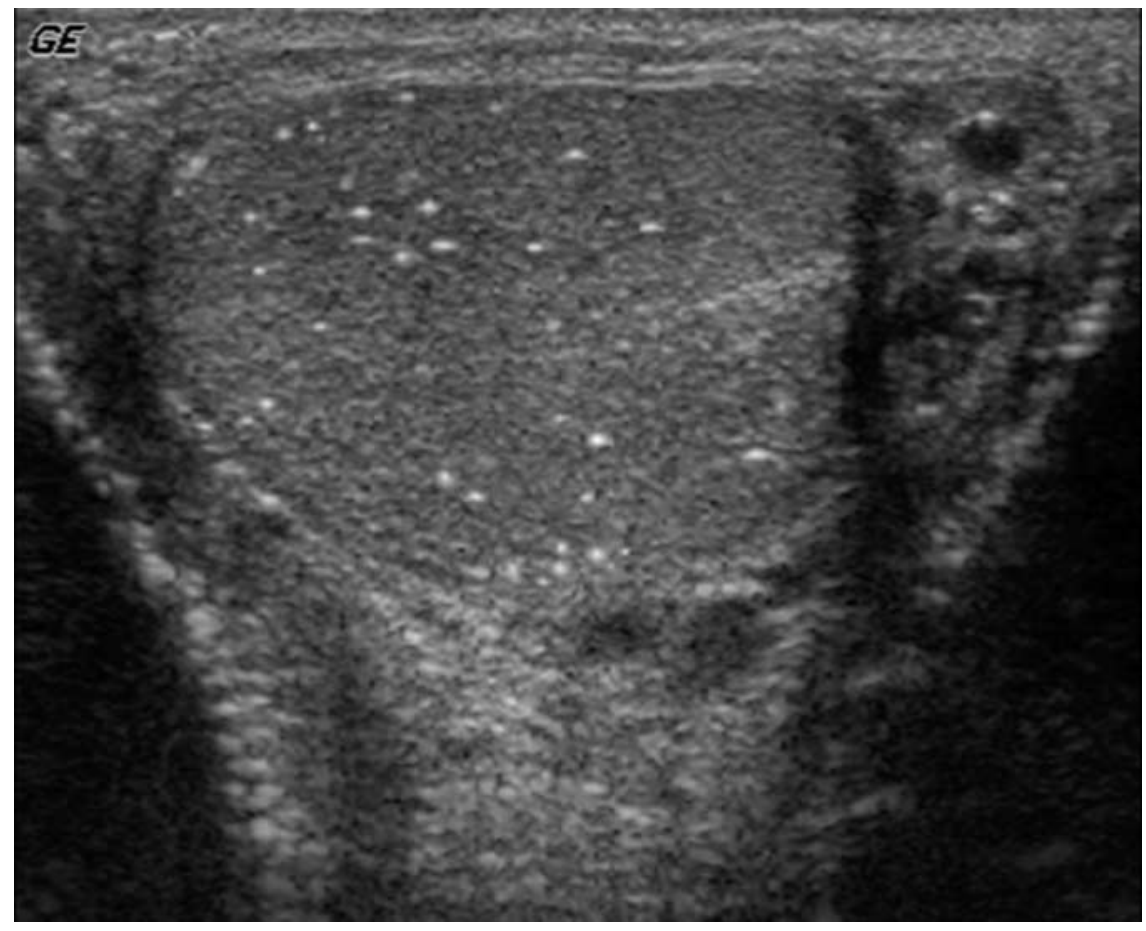

Figure 7 Ultrasonographic picture of microlithiasis in a testicle. The white spots represent microcalcifications. 
Table 3 Total testosterone levels in relation to treatment given and time at follow-up.

\begin{tabular}{lllllll}
\hline & \multicolumn{5}{c}{ Median - testosterone - nmol/l $(n)$ range } \\
\cline { 2 - 7 } & T0 & T6 & T12 & T24 & T36 & T60 \\
\hline Surveillance & $17(3)$ & $9.8(7)$ & $10(5)$ & $11(4)$ & $9.8(4)$ & $9.5(4)$ \\
& $11-22$ & $6.4-15$ & $6.8-14$ & $9.6-14$ & $8.4-16$ & $6.7-22$ \\
ACT & $14(26)$ & $14(24)$ & $14(25)$ & $14(27)$ & $13(24)$ & $12(14)$ \\
& $6.7-23$ & $10-22$ & $7.7-21$ & $9.1-24$ & $8.2-22$ & $6.3-20$ \\
HCT & $15(20)$ & $13(18)$ & $10(17)$ & $13(18)$ & $14(19)$ & $14(20)$ \\
RT & $4.0-29$ & $1.5-22$ & $5.4-21$ & $4.4-23$ & $4.8-33$ & $7.9-21$ \\
HDT & $12(18)$ & $12(19)$ & $12(23)$ & $12(17)$ & $11(14)$ & $13(17)$ \\
All patients & $7.5-20$ & $8.1-21$ & $6.9-24$ & $7.1-18$ & $1.3-23$ & $3.0-18$ \\
& $13(4)$ & $15(4)$ & $11(5)$ & $10(5)$ & $10(4)$ & $7.8(1)$ \\
& $12-18$ & $9.1-33$ & $8.2-43$ & $6.8-12$ & $10-13$ & 7.8 \\
\end{tabular}

$\mathrm{ACT}$, one to two cycles of adjuvant chemotherapy; HCT, three to four cycles of chemotherapy; HDT, more intense treatment. More than four cycles of chemotherapy; RT, adjuvant radiotherapy; $n$, amount of patients.

The signs of hypogonadism are non-characteristic and good biochemical markers of androgen deficiency are lacking. The clinical diagnosis is usually based on the combination of symptoms and serum levels of testosterone and LH. In this study, we have only used biochemical parameters in defining hypogonadism. The levels of $10 \mathrm{nmol} / \mathrm{l}$ for total testosterone and $10 \mathrm{IU} / \mathrm{l}$ for $\mathrm{LH}$ are generally accepted as useful markers of hypogonadism in younger males (22). A shortcoming in current study is the change in the methods for testosterone and LH measurements. However, conversion of the results from the method used in the first part of the study to the one applied during the second part was based on more than 30 subjects tested with both methods. Furthermore, no statistically significant difference between measurements performed with the different methods was found at any time point. Finally, the type of method applied for hormone measurement was included as confounding factor in the statistical analysis. We therefore believe that our results are reliable despite the methodological drawback.

Blood samples for hormone analysis were collected between 0900 and $1500 \mathrm{~h}$. Due to the diurnal variation in testosterone concentration, sampling should ideally be performed before $1000 \mathrm{~h}$. However, the time of blood sampling was randomly distributed among the patients and not related to any of the possible predictors of hypogonadism tested. Furthermore, for all statistically significant associations, same trends were found when LH > $10 \mathrm{IU} / \mathrm{l}$ was used as the only indicator of hypogonadism. Unlike testosterone, LH does not decrease during the daytime.

Although all subjects with increased post-orchidectomy hCG were excluded from the T0 analysis, we did not have access to the information regarding increased preorchidectomy hCG normalizing following removal of the tumour-bearing testis. Due to the length of the time period between surgery and initiation of adjuvant treatment (at least 6 weeks), we do not expect that testosterone and LH levels were influenced in these men by previous hCG elevation. However, any reminiscent hCG effect would tend to change to classification of a subject at TO from hypo- to normogonadal and, therefore, rather reduce the power of hypogonadism at $\mathrm{T} 0$ as predictor of post-treatment androgen deficiency.

Table 4 Total luteinizing hormone (LH) levels in relation to treatment given and time at follow-up.

\begin{tabular}{lllllll}
\hline & \multicolumn{5}{l}{ Median - LH values - IU/I $(n)$ range } \\
\cline { 2 - 7 } & T0 & T6 & T12 & T24 & T36 & T60 \\
\hline Surveillance & $5.9(3)$ & $7.2(7)$ & $5.5(5)$ & $5.2(4)$ & $5.3(4)$ & $5.0(4)$ \\
ACT & $5.5-11$ & $4.0-18$ & $4.0-14$ & $2.8-7.8$ & $4.0-6.0$ & $3.0-9.0$ \\
& $5.6(25)$ & $6.7(25)$ & $6.6(24)$ & $5.8(27)$ & $6.6(23)$ & $5.8(14)$ \\
HCT & $1.4-15$ & $2.2-19$ & $2.4-25$ & $2.0-15$ & $3.0-17$ & $3.0-11$ \\
& $3.8(20)$ & $12(18)$ & $11(19)$ & $7.8(18)$ & $6.3(19)$ & $5.0(20)$ \\
RT & $0.1-67$ & $5.4-33$ & $2.5-24$ & $0.3-23$ & $0-22$ & $2.0-12$ \\
HDT & $5.2(18)$ & $8.4(18)$ & $6.6(22)$ & $5.3(17)$ & $5.4(14)$ & $5.2(17)$ \\
All patients & $1.6-21$ & $0.2-30$ & $2.5-30$ & $3.4-31$ & $0-12$ & $0-16$ \\
& $6.0(4)$ & $13(4)$ & $12(4)$ & $10(5)$ & $7.6(4)$ & $13(1)$ \\
& $0.2-12$ & $7.0-23$ & $7.2-27$ & $1.9-21$ & $6.0-14$ & 13 \\
\end{tabular}

$\mathrm{ACT}$, one to two cycles of adjuvant chemotherapy; HCT, three to four cycles of chemotherapy; HDT, more intense treatment. More than four cycles of chemotherapy; RT, adjuvant radiotherapy; $n$, amount of patients. 
Finally, it should be kept in mind that for some of the analyses the number of subjects included was relatively low and lack of statistical significance due to a type 2 error cannot be excluded.

In summary, men presenting with TGCC have an increased risk of androgen deficiency (29) but the risk of developing hypogonadism has been more or less overseen in this category of patients. Measuring of testosterone and $\mathrm{LH}$ concentration is not a general clinical routine during the follow-up of TGCC men. The identification of risk factors for development of $\mathrm{BH}$ is, therefore, important for proper management of these patients. Current study shows that $\mathrm{BH}$ prior to oncological treatment and in those presenting with microlithiasis by ultrasound are strong predictors of subsequent hypogonadism. Three to four cycles of chemotherapy and adjuvant radiotherapy also implies an increased risk of being hypogonadal, at least during the first post-treatment year. This should be taken into consideration in the pre-treatment investigation and follow-up of men treated for TGCC.

\section{Acknowledgements}

We thank Katarina Jepson and Camilla Anderberg for excellent technical assistance, Annette Möller for helping us with organizing the patient flow. We also thank Nils Göran Persson, MD at the Dept of Clinical Chemistry, Malmö and Anders Isaksson, MD at the Dept of Clinical Chemistry, Lund for helping us with the calculation of conversion factors between the different hormone analyses and Anna Rignell-Hydbom for providing us with serum samples and data for performing such comparison. Finally, we also want to thank Per Flodgren, MD at the Dept of Oncology, Lund for helping us with the inclusion of patients in the study. The study was supported by grants from Swedish Governmental Funding for Clinical Research, the Swedish Cancer Society (Grant No. 4423), Gunnar Nilssons Cancerstiftelse, Swedish Childhood Cancer Society (Grant Nos 05/056 and RKT04/001), Malmö University Hospital Foundation for Cancer Research, and Foundation for Urological Research.

\section{References}

1 Nijman JM, Schraffordt Koops H, Kremer J \& Sleijfer DT. Gonadal function after surgery and chemotherapy in men with stage II and III nonseminomatous testicular tumors. Journal of Clinical Oncology $19875651-656$.

2 Willemse PHB, Sleijfer DT, Sluiter WJ, Schraffordt Koops H \& Doorenbos H. Altered Leydig cell function in patients with testicular cancer: evidence for a bilateral testicular defect. Acta Endocrinologica 1983102 616-624.

3 Fossa SD, Abyholm T \& Aakvaag A. Spermatogenesis and hormonal status after orchiectomy for cancer and before supplementary treatment. European Urology 1984 10 173-177.

4 Petersen PM, Skakkebæk NE, Rørth M \& Giwercman A. Semen quality and reproductive hormones before and after orchiectomy in men with testicular cancer. Journal of Urology $1999161822-826$.
5 Fossa SD, Lehne G, Heimdal K \& Theodorsen L. Clinical and biochemical long-term toxicity after postoperative cisplatin-based chemotherapy in patients with low-stage testicular cancer. Oncology 199552 300-305.

6 Hansen PV \& Hansen SW. Gonadal function in men with testicular germ cell cancer: the influence of cisplatin-based chemotherapy. European Urology 199323 153-156.

7 Bissett D, Kunkeler L, Zwanenburg L, Paul J, Gray C, Swan IRC, Kerr DJ \& Kaye SB. Long-term sequelae of treatment for testicular germ cell tumours. British Journal of Cancer 199062 655-659.

8 Strumberg D, Brugge S, Korn MW, Koeppen S, Ranft J, Scheiber G, Reiners C, Mockel C, Seeber S \& Scheulen ME. Evaluation of longterm toxicity in patients after cisplatin-based chemotherapy for non-seminomatous testicular cancer. Annals of Oncology 200213 229-236.

9 Petersen PM, Hansen SW, Giwercman A, Rørth M \& Skakkebæk NE. Dose-dependent impairment of testicular function in patients treated with cisplatin-based chemotherapy for germ cell cancer. Annals of Oncology 19945 355-358.

10 Fosså SD, Ous S, Åbyholm T, Norman N \& Loeb M. Post-treatment fertility in patients with testicular cancer. British Journal of Urology 198557 210-214.

11 Nord C, Bjoro T, Ellingsen D, Mykletun A, Dahl O, Klepp O, Bremnes RM, Wist E \& Fossa SD. Gonadal hormones in long-term survivors 10 years after treatment for unilateral testicular cancer. European Urology 200344 322-328.

12 Huddart RA, Norman A, Moynihan C, Horwich A, Parker C, Nicholls E \& Dearnaley DP. Fertility, gonadal and sexual function in survivors of testicular cancer. British Journal of Cancer 200593 200-207.

13 Tut TG, Ghadessy FJ, Trifiro MA, Pinsky L \& Young EL. Long polyglutamine tracts in the androgen receptor are associated with reduced trans-activation, impaired sperm production, and male infertility. Journal of Clinical Endocrinology and Metabolism 199782 3777-3782.

14 Lundin KB, Giwercman YL, Rylander L, Hagmar L \& Giwercman A. Androgen receptor gene GGN repeat length and reproductive characteristics in young Swedish men. European Journal of Endocrinology 2006155 347-354.

15 Eberhard J, Stahl O, Giwercman Y, Cwikiel M, Cavallin-Stahl E, Lundin KB, Flodgren P \& Giwercman A. Impact of therapy and androgen receptor polymorphism on sperm concentration in men treated for testicular germ cell cancer: a longitudinal study. Human Reproduction 200419 1418-1425.

16 Klepp O, Dahl O, Flodgren P, Stierner U, Olsson AM, Oldbring J, Nielsson S, Daehlin L, Tornblom M, Smaland R, Starkhammar H, Abramsson L, Wist E, Raabe N, Edekling T \& Cavallin-Stahl E. Risk-adapted treatment of clinical stage I non-seminoma testis cancer. European Journal of Cancer 19977 1038-1044.

17 Laguna MP, Pizzocaro G, Klepp O, Algaba F, Kisbenedek L \& Leiva O. EAU guidelines on testicular cancer. European Urology 200140 102-110.

18 Lundin KB, Giwercman A, Richthoff J, Abrahamsson P-A \& Giwercman YL. No association between mutations in the human androgen receptor GGN repeat and inter-sex conditions. Molecular Human Reproduction 20039 375-379.

19 Lenz S, Giwercman A, Skakkebæk NE, Bruun E \& FrimodtMøller C. Ultrasound in detection of early neoplasia of the testis. International Journal of Andrology 198710 187-190.

20 von Eckardstein S, Tsakmakidis G, Kamischke A \& Nieschlag E. Sonographic testicular microlithiasis as an indicator of premalignant conditions in normal and infertile men. Journal of Andrology 200122 818-824.

21 Lenz S, Giwercman A, Elsborg A, Cohr K-H \& Jelnes JE. Ultrasonic testicular texture and size in 444 men from the general population: correlation to semen quality. European Urology 1993 24 231-238.

22 Nieschlag E, Behre HM, Bouchard P, Corrales JJ, Jones TH, Stalla GK, Webb SM \& Wu FC. Testosterone replacement therapy: current trends and future directions. Human Reproduction Update $200410409-419$. 
23 Hansen SW, Berthelsen JG \& von der Maase H. Long-term fertility and Leydig cell function in patients treated for germ cell cancer with cisplatin, vinblastine, and bleomycin versus surveillance. Journal of Clinical Oncology 19908 1695-1698.

24 Palmieri G, Lotrecchiano G, Ricci G, Spiezia R, Lombardi G, Bianco AR \& Torino G. Gonadal function after multimodality treatment in men with testicular germ cell cancer. European Journal of Endocrinology 1996134 431-436.

25 Brennemann W, Stoffel-Wagner B, Helmers A, Mezger J, Jager N \& Klingmuller D. Gonadal function of patients treated with cisplatin based chemotherapy for germ cell cancer. Journal of Urology 1997 158 844-850.

26 Hoei-Hansen CE, Sommer P, Meyts ER \& Skakkebaek NE. A rare diagnosis: testicular dysgenesis with carcinoma in situ detected in a patient with ultrasonic microlithiasis. Asian Journal of Andrology $20057445-447$.
27 Bach AM, Hann LE, Hadar O, Shi W, Yoo HH, Giess CS, Sheinfeld J \& Thaler H. Testicular microlithiasis: what is its association with testicular cancer? Radiology 2001220 70-75.

28 Giwercman YL, Richthoff J, Lilja H, Anderberg C, Abrahamsson PA \& Giwercman A. Androgen receptor CAG repeat length correlates with semen PSA levels in adolescence. Prostate 200459 227-233.

29 Petersen PM, Skakkebæk NE \& Giwercman A. Gonadal function in men with testicular cancer: biological and clinical aspects. Acta Pathologica, Microbiologica, et Immunologica Scandinavica 1998106 24-36.

30 Dearnaley D, Huddart R \& Horwich A. Regular review: managing testicular cancer. BMJ 2001322 1583-1588.

Received 23 December 2007

Accepted 20 January 2008 